\title{
Sporadic Hemangioblastoma of the Cavernous Sinus and Meckel's Cave
}

\author{
Ali Kord Valeshabad ${ }^{1} \quad$ Lekui Xiao $^{1} \quad$ Sepideh Amin-Hanjani ${ }^{2} \quad$ Alaa Alsadi $^{3}$ Tibor Valyi-Nagy ${ }^{3} \quad$ jinsuh Kim $^{4}$ \\ ${ }^{1}$ Department of Radiology, University of Illinois at Chicago, Chicago, \\ Illinois, United States \\ 2 Department of Neurosurgery, University of Illinois at Chicago, \\ Chicago, Illinois, United States \\ ${ }^{3}$ Department of Pathology, University of Illinois at Chicago, Chicago, \\ Illinois, United States \\ ${ }^{4}$ Department of Radiology, University of Alabama at Birmingham, \\ Birmingham, Alabama, United States \\ Address for correspondence Jinsuh Kim, MD, MS, Department of \\ Radiology, University of Alabama at Birmingham, 619 South 19th \\ street, Birmingham, AL 35249, United States \\ (e-mail: jinsuhkim@uabmc.edu).
}

J Neurol Surg Rep 2018;79:e98-e102.

\begin{abstract}
\section{Keywords}

- hemangioblastoma

- cavernous sinus

- Meckel's cave

- cerebellopontine angle

- denervation atrophy

Objectives To describe an extremely rare case of sporadic hemangioblastoma (HB) within the cavernous sinus and Meckel's cave with extension to the cerebellopontine angle (CPA) cistern.

Methods A 73-year-old male presented with hearing loss, unilateral ptosis, and facial numbness.

Results The imaging showed a complex cystic-solid mass centered at the left cavernous sinus and Meckel's cave with extension to the CPA cistern. Patient underwent retrosigmoid craniectomy for partial resection of the CPA angle component of the mass. Surgical pathology confirmed the diagnosis of $\mathrm{HB}$ and patient was scheduled for subsequent radiotherapy of the residual mass.

Conclusions We present an exceptional case of supratentorial HB without associated von Hippel-Lindau (VHL) disease, which was predominantly located in the cavernous sinus and Meckel's cave and led to multiple cranial nerve symptoms. We describe imaging characteristics and radiologic-pathologic correlation of this atypically located $\mathrm{HB}$, which can be difficult to consider in the differential diagnosis presurgically.
\end{abstract}

\section{Introduction}

Hemangioblastoma (HB) is a rare, slow-growing and lowgrade vascular tumor which accounts for 1 to $2.5 \%$ of intracranial tumors and $4 \%$ of spinal cord tumors. HB is typically located in the posterior fossa vascular origin tumor, comprising of 7 to $10 \%$ of all posterior fossa tumors. ${ }^{1,2}$ There have been previous reports on uncommon locations of $\mathrm{HB}$, such as the cerebellopontine angle (CPA) cistern, sella turcica, and supratentorial brain parenchyma. ${ }^{3-6}$ These unusual presentations of $\mathrm{HB}$ engenders a diagnostic challenge to distinguish it from far more common tumors, such as vestibular schwannoma and meningioma. ${ }^{1,7}$ However, HB primarily involving the cavernous sinus that caused

received

February 13, 2018

accepted

August 28, 2018
DOI https://doi.org/

10.1055/s-0038-1676455. ISSN 2193-6358. denervation atrophy of the masticator space muscles has not been described in previous literatures. In this case report, we present an unusual case of sporadic HB centered within the cavernous sinus and Meckel's cave with hemorrhagic extension to the CPA cistern, causing III, V, and VIII cranial neuropathies with associated denervation atrophy of the masticator muscles.

\section{Case}

A 73-year-old male with history of hypothyroidism and Asperger's syndrome presented to an outside hospital for evaluation of erratic driving. Admission noncontrast head computed tomography (CT) exam demonstrated a $5 \times 3 \mathrm{~cm}$ extra-axial (c) 2018 Georg Thieme Verlag KG
Stuttgart · New York

License terms

$\Theta(1) \Theta \Theta$ 


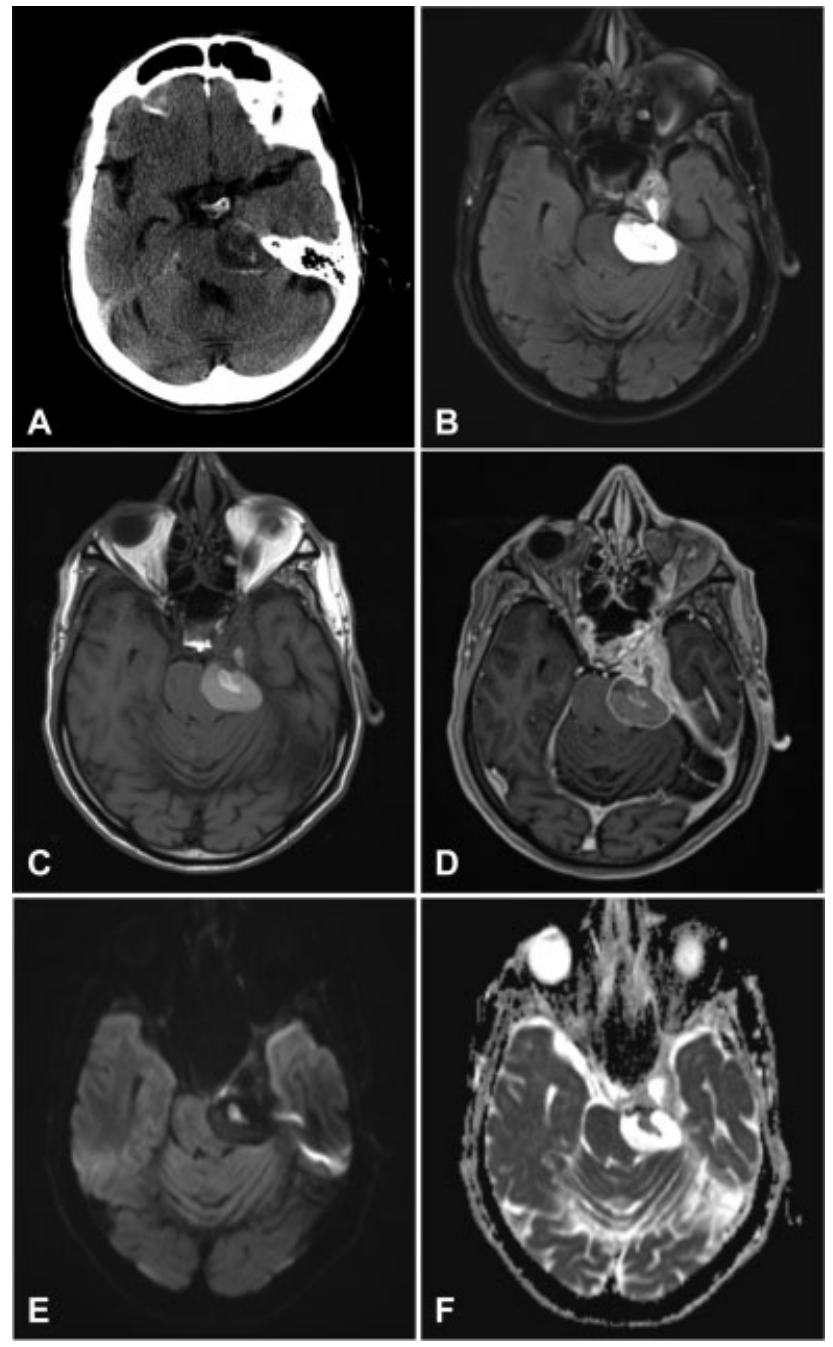

Fig. 1 Preoperative axial (A) computed tomography (CT) of the head shows a complex mass within the left cavernous sinus and CPA cistern compressing the left pons. Magnetic resonance imaging (MRI) (C-E) reveals a solid and cystic mass centered at the cavernous sinus and Meckel's cave with a bulky hemorrhagic extension to the CPA cistern. The main mass is isointense on T1-weighted images (C) and heterogeneously isointense on T2-weighted images (B) with multilocular small cysts containing hemorrhagic layering. The solid component of the lesion is avidly enhancing on postcontrast images (D). Signal of diffusion-weighted sequence imaging is lower than the brain parenchyma (E) with ADC value of $1,300 \pm 643\left(\times 10^{-6} \mathrm{~mm}^{2} / \mathrm{s} ; \mathrm{F}\right)$. CPA, cerebellopontine angle. ADC, apparent diffusion coefficient.

mass with heterogeneous hypodensity located in the left cavernous sinus and the CPA cistern, significantly compressing the ipsilateral pons ( $\mathbf{- F i g . 1}$ ). Subsequent magnetic resonance imaging (MRI; - Fig. 1) revealed a solid and cystic mass centered at the cavernous sinus and Meckel's cave with a bulky hemorrhagic extension to the CPA cistern. MRI signal characteristics of the main mass were isointensity on T1-weighted images and heterogeneous isointensity solid component with multilocular small cysts containing hemorrhagic layering on T2-weighted images, as well as avid enhancement on contrastenhanced T1-weighed scans. Signal of diffusion-weighted sequence imaging was lower than the brain parenchyma with apparent diffusion coefficient (ADC) value of
$1,300 \pm 643\left(\times 10^{-6} \mathrm{~mm}^{2} / \mathrm{s}\right)$. The mass incompletely encased and medially displaced the cavernous portion of the left internal carotid artery. The mass exhibited inferolateral extension into the ipsilateral foramen ovale and a nodular projection to the vicinity of the optic tract superiorly. Typical imaging characteristics of $\mathrm{HB}$, such as a mural nodule within CSF signal cyst and serpentine flow voids were absent. In addition, there was a dural tail extension into the superior porus acusticus as well as ipsilateral tentorial margin (not shown in the figures). As sequelae of mandibular division of the trigeminal nerve (V3) involvement by the tumor, chronic denervation atrophy of the medial and lateral pterygoid, masseter and temporalis muscles, and atrophy of the parotid gland were evident on the left side (-Fig. 2A). Additionally, the fundus of the right internal auditory canal demonstrated a nodular mass with signal characteristics similar with the left cavernous sinus mass measuring $8 \mathrm{~mm}$ in length (not shown in the figures). Differential diagnostic consideration included a solitary fibrous tumor (hemangioperycytoma), atypical meningioma, trigeminal schwannoma, cavernous hemangioma, and metastatic tumor.

On admission at our institution, physical exam was significant for decreased hearing predominatntly on the right and decreased sensation to touch in the territory of the ophthalmic (V1) and maxillary (V2) divisions of the left trigeminal nerve. Left ptosis was noted which was present for an undetermined period of time. The patient had a chronic history of slow and circumlocutory speech. Electroencephalography was performed due to concern for partial seizure and demonstrated sharp and spike-wave discharges consistent with left temporal onset epilepsy. The patient was started on medication for seizure prophylaxis. Laboratory tests were within the normal limits without polycythemia in particular. Given the mass effect in the posterior fossa, the patient underwent a left retrosigmoid craniotomy for resection of the left CPA component of the mass, both to provide a diagnosis and decompress the brainstem mass effect. At the time of surgery, a cystic lesion with a thick capsule and solid and moderately vascular component adhering the trigeminal nerve was encountered and removed in piecemeal fashion; the gross appearance was not typical for usual parenchymal HBs encountered in the posterior fossa which are often recognizable by their orange hue and extreme vascularity precluding piecemeal resection. Postoperative MRI demonstrated complete resection of the predominantly hemorrhagic portion of the tumor in the left prepontine cistern and CPA, with persistent tumor bulk residing in the left cavernous sinus and Meckel's cave. Pathologic study of the surgical specimen ( - Fig. 3 ) showed a cystic-solid lesion containing mural nodules which was an admixture of vascular structures and stromal proliferation. The stromal proliferation was positive for vimentin, carbonic anhydrase IX, and focally positive for inhibin. The vascular structures were highlighted by CD31. The findings were compatible with a final diagnosis of grade 1 hemangioblastoma.

One-month follow-up at neurosurgery clinic showed baseline left sided hearing difficulties and left trigeminal nerve paresthesia which was slowly improving. There was no significant change in the mild baseline ptosis and no new 

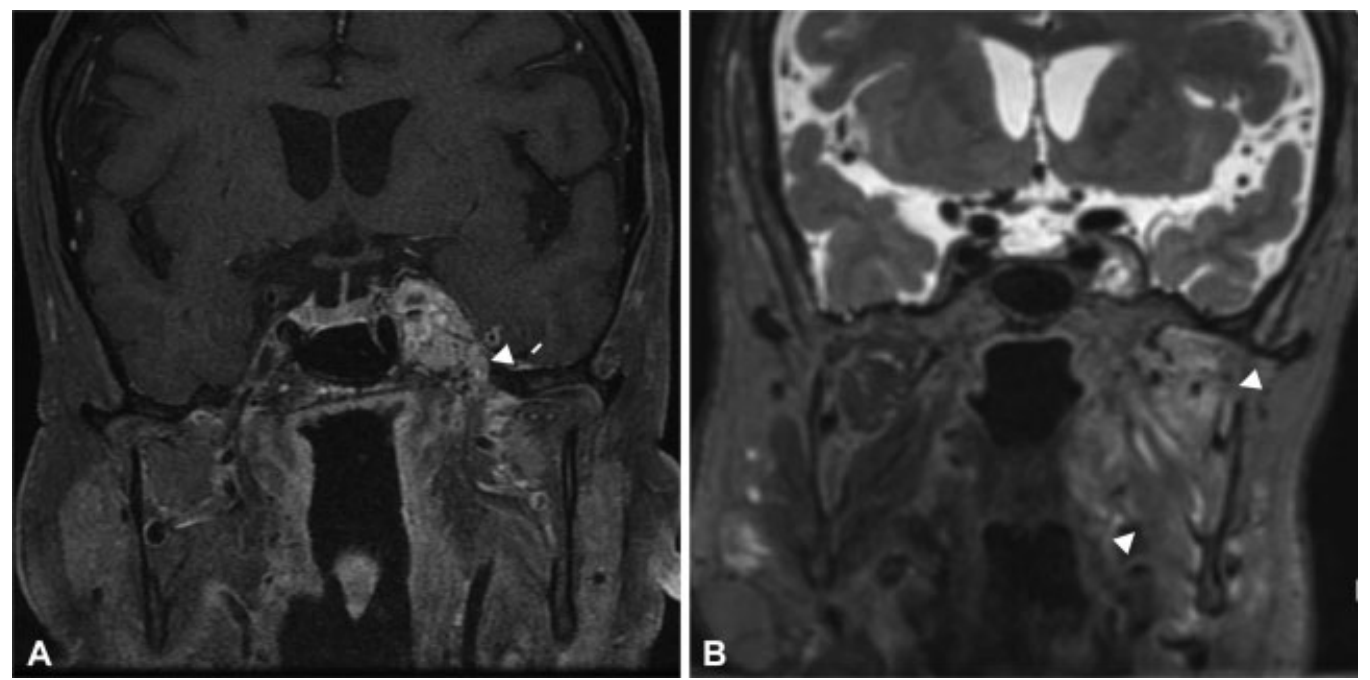

Fig. 2 Postoperative coronal T1-weighted image with fat saturation (A) demonstrates typical imaging characteristics of HB with serpentine flow voids. The mass incompletely encases and medially displaces the cavernous portion of the left internal carotid artery (A). The mass exhibits inferolateral extension into the ipsilateral foramen ovale (white arrow; A) and a nodular projection to the vicinity of the optic tract superiorly. Coronal T2-weighted image (B) reveals the sequelae of CN V3 involvement by the tumor with advanced chronic denervation atrophy with fatty replacement of the left medial and lateral pterygoid, masseter and temporalis muscles (white arrowheads; B). CN, cranial nerve; HB, hemangioblastoma.

neurological deficits. Three-month postoperative MRI showed slightly increased size of the residual mass with increased multilocular cystic component and newly visualized multiple flow voids of enlarged feeding vessels (-Fig. 2B). The mass in the right internal auditory canal, presumed to be a synchronous vestibular schwannoma versus second $\mathrm{HB}$, was persistently identified without gross interval change. The patient was scheduled for subsequent radiotherapy of the residual cavernous sinus mass to prevent future growth.

\section{Discussion}

HBs usually involve young-to-middle-aged adults, with a male predilection and a male-female ratio of 1.3-2.1:1. They either develop sporadically, approximately $75 \%$ of cases, or in association with VHL disease, with 60 to $85 \%$ of these patients having HB. Due to their slow growth, patients remain asymptomatic for a long time and symptoms manifest depending on tumor size. Patients most commonly present with headache, followed by symptoms of raised intracranial pressure, cerebellar dysfunction, and altered mental status. In $20 \%$ of cases, polycythemia can be seen due to overproduction of erythropoietin. ${ }^{1,2}$ The patient in this study presented with cranial neuropathies affecting cranial nerves III, V, and VIII due to the location of the mass. Patients with CPA HB, similarly to other CPA tumors, often present with unilateral inner ear symptoms including hearing loss, tinnitus, vertigo, balance issues, and facial paresthesia which make it challenging to distinguish this tumor type based on clinical symptomatology.

The most common imaging presentation of $\mathrm{HB}$ is an intraaxial mass with cyst and enhancing mural nodule in the vicinity of the pial surface of the posterior fossa structure. ${ }^{8}$ While 50 to $60 \%$ of HBs are cystic with a mural nodule, $40 \%$ are solid at presentation. Unenhanced CT typically demonstrates a lowdensity cyst which is isodense to the hyperdense nodule without calcification. ${ }^{1}$ On contrast-enhanced $\mathrm{CT}$, the nodule enhances uniformly without enhancement of the cyst wall. CT angiogram may show arterial feeders of the lesion. Basic MRI characteristics include the cystic component of the tumor as usually $\mathrm{T} 1$ hypointense and $\mathrm{T} 2$ hyperintense similar to cerebrospinal fluid. ${ }^{1}$ The nodule is usually isointense on both $\mathrm{T} 1$ and T2 and enhances strongly after contrast. However, low signal of diffusion weighted imaging (DWI) with increased ADC of the solid components in HBs is not commonly observed. Furthermore, the vascularity of $\mathrm{HB}$ can be recognized with flow voids of dilated feeding arteries on MRI and prolonged tumor blush on angiography. ${ }^{6,7}$ In our case, the mass was found in an uncustomary location of the cavernous sinus and Meckel's cave with extension to the ipsilateral CPA cistern leading to denervation atrophy of the muscles in the masticator space secondary to damage of the third (mandibular) branch of the $\mathrm{CN} \mathrm{V}$ (cranial nerve). In addition, left sided neuropathies of $\mathrm{CN}$ III, and first, and second branches (V1 and V2) of V were found on physical examination in our patient which were well correlated with $\mathrm{CN}$ anatomy and imaging localization of the tumor.

HBs can be macroscopically divided into solid and more common cystic subtypes. Gross appearance is an orange-red, well-circumscribed, nonencapsulated, and highly vascular mass that abuts the leptomeninges. ${ }^{1}$ It may be associated with a cyst that contains a yellow-brown fluid. Histologically, HBs can be divided into cellular and reticular subtypes. The atypical gross appearance in this case may reflect degeneration of the CPA component of the tumor resulting in a hemorrhagic cyst with a thick capsule.

The cellular characteristics of HB are foamy stromal cells clustered in sheets about tangles of capillaries whereas the cells are distributed evenly around a density of capillaries in the reticular subtype. These neoplastic cells stain positively 

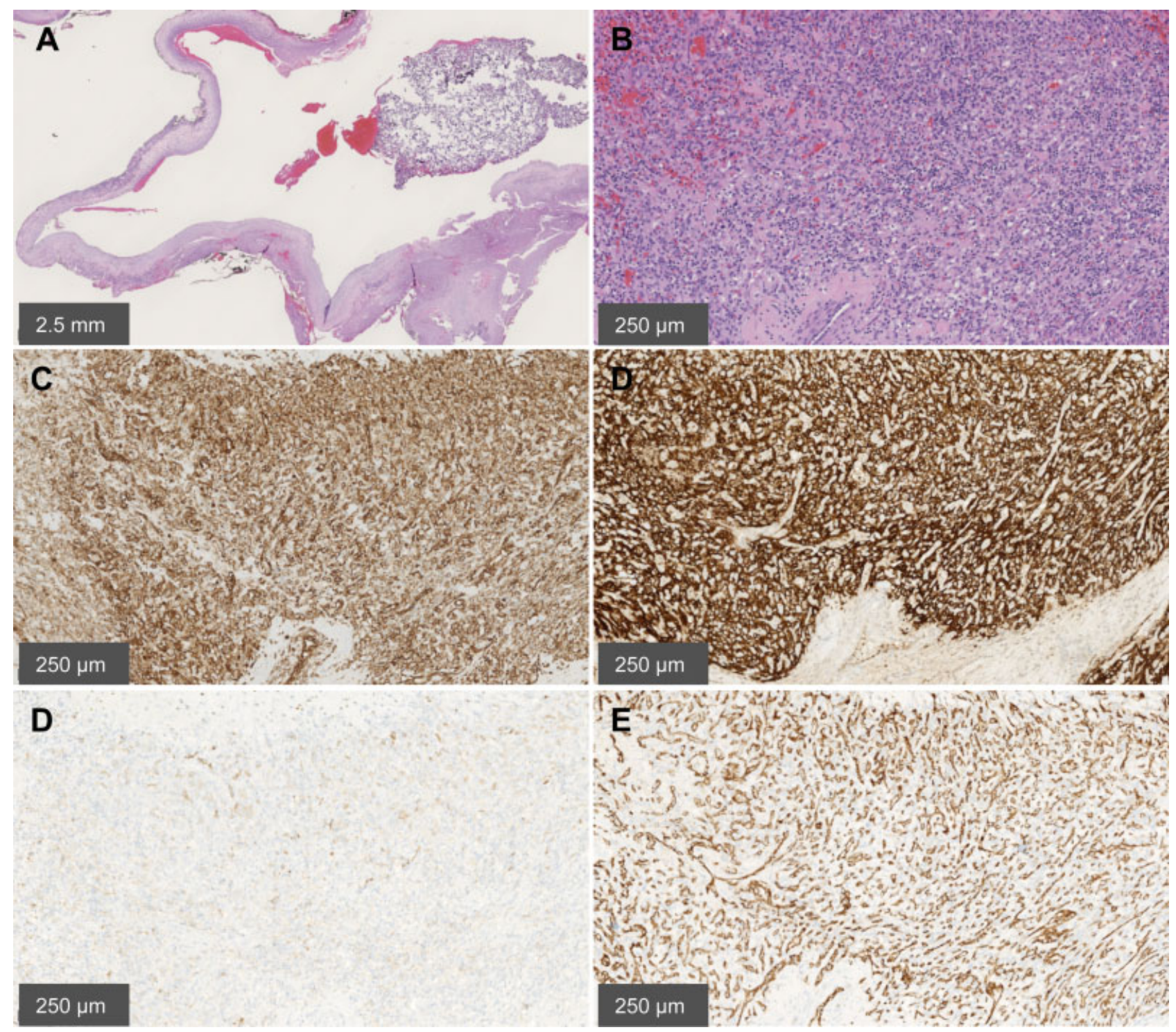

Fig. 3 Pathologic study of the surgical specimen. (A) Low magnification shows a cystic nature of the lesion; haematoxylin and eosin $\times 10$. (B) Higher magnification demonstrates the dual component tumor of hemorrhagic, thin-walled, vascular channels lined by flattened endothelium (especially to the left of the picture) and of stromal proliferation with large, polygonal, cells containing vacuolated cytoplasm (especially mid and proximal right of the picture). Note the prominent mast cell infiltrate (small, dark, round nuclei); haematoxylin and eosin $\times 10$. (C) Tumor cells are strongly positive for vimentin. Vimentin stain $\times 100$. (D) Tumor cells are strongly positive for carbonic anhydrase IX; CA IX stain $\times 100$. (E) Tumor cells are focally positive for inhibin; Inhibin stain $\times 100$. (F) Vascular channels are highlighted by CD31; CD31 stain $\times 100$. CA, carbonic anhydrase.

for inhibin A, GLUT-1, vimentin, and S100, in addition to overexpressing the vascular endothelial growth factor (VEGF) protein. ${ }^{9}$ The surgical specimen, in this study, demonstrated a highly vascular cystic-solid lesion which was positive for vimentin, carbonic anhydrase IX, inhibin, and CD31, most compatible with HB.

HBs are usually treated using en bloc surgical resection as piecemeal can result in catastrophic hemorrhage. ${ }^{7,10}$ Imaging modalities play an essential role for surgical and medical planning of patients with HBs. Tumor characteristics observed on preoperative images of sporadic cerebellar HBs have been reported as one of the most important clinical factors correlated with both immediate and long-term outcomes after surgery. ${ }^{11}$ Patients with solid cerebellar HBs are reported to have more poor outcomes compared to patients with cystic tumors. ${ }^{11,12}$ In addition, tumor size, location, and vascularity seen on presurgical imaging are important factors to assess the need for adjuvant therapy, such as preoperative embolization. ${ }^{13}$ Small tumors which are stable and asymptomatic can be managed expectantly. For patients with VHL, it is recommended to screen the entire CNS (central nervous system) for HBs. Two-third of those with a single VHL-associated HB will develop additional lesions at an average of one lesion every 2 years. ${ }^{10}$ The patient in this study was scheduled for subsequent radiotherapy the initial resection. The 10 year survival rate for surgical treatment of HBs has been reported up to $85 \%$ with a recurrence rate of 15 to $20 \%{ }^{14}$

In summary, a rare sporadic supratentorial HB involving the cavernous sinus and Meckel's cave with extension to the CPA cistern is reported here which has not been described in 
the previous literature to our knowledge. Presurgical imaging diagnosis of the intracranial tumor, especially involving skull base, is an important part of care to adequately assist surgical planning and establish the follow-up treatment strategy. However, HB in odd locations can be diagnostically challenging, which may lead to misinterpretation of MRI findings, and potentially preclude optimal surgical planning. Therefore, imaging diagnosticians should be aware of unusual manifestation of sporadic HB that mimics other common extra-axial tumors.

\section{Conflict of Interest \\ None declared.}

\section{References}

1 Ho VB, Smirniotopoulos JG, Murphy FM, Rushing EJ. Radiologicpathologic correlation: hemangioblastoma. AJNR Am J Neuroradiol 1992;13(05):1343-1352

2 Liao CC, Huang YH. Clinical features and surgical outcomes of sporadic cerebellar hemangioblastomas. Clin Neurol Neurosurg 2014;125:160-165

3 Bush ML, Pritchett C, Packer M, Ray-Chaudhury A, Jacob A. Hemangioblastoma of the cerebellopontine angle. Arch Otolaryngol Head Neck Surg 2010;136(07):734-738

4 Mills SA, Oh MC, Rutkowski MJ, Sughrue ME, Barani IJ, Parsa AT. Supratentorial hemangioblastoma: clinical features, prognosis, and predictive value of location for von Hippel-Lindau disease. Neuro-oncol 2012;14(08):1097-1104
5 Moon BH, Park SK, Han Y-M. Large solid hemangioblastoma in the cerebellopontine angle: complete resection using the transcondylar fossa approach. Brain Tumor Res Treat 2014;2(02):128-131

6 Amelot A, Bouazza S, Polivka M, George B, Bresson D. Sporadically second localization of cerebellar hemangioblastoma in sella turcica mimicking a meningioma with no associated von Hippel-Lindau disease. Br J Neurosurg 2015;29(04):589-591

7 Rachinger J, Buslei R, Prell J, Strauss C. Solid haemangioblastomas of the CNS: a review of 17 consecutive cases. Neurosurg Rev 2009; 32(01):37-47, discussion 47-48

8 Raz E, Zagzag D, Saba L, et al. Cyst with a mural nodule tumor of the brain. Cancer Imaging 2012;12:237-244

9 Takeuchi H, Hashimoto N, Kitai R, Kubota T. A report of supratentorial leptomeningeal hemangioblastoma and a literature review. Neuropathology 2008;28(01):98-102

10 Conway JE, Chou D, Clatterbuck RE, Brem H, Long DM, Rigamonti D. Hemangioblastomas of the central nervous system in von Hippel-Lindau syndrome and sporadic disease. Neurosurgery 2001;48(01):55-62, discussion 62-63

11 Fukuda M, Takao T, Hiraishi T, et al. Clinical factors predicting outcomes after surgical resection for sporadic cerebellar hemangioblastomas. World Neurosurg 2014;82(05):815-821

12 Karabagli H, Genc A, Karabagli P, Abacioglu U, Seker A, Kilic T. Outcomes of gamma knife treatment for solid intracranial hemangioblastomas. J Clin Neurosci 2010;17(06):706-710

13 Shin GW, Jeong HW, Seo JH, Kim ST, Choo HJ, Lee SJ. Preoperative embolization of cerebellar hemangioblastoma with onyx: report of three cases. Neurointervention 2014;9(01):45-49

14 Kano H, Shuto T, Iwai Y, et al. Stereotactic radiosurgery for intracranial hemangioblastomas: a retrospective international outcome study. J Neurosurg 2015;122(06):1469-1478 